\title{
Location, Spreading and Oral Corticosteroids are Associated with Insomnia in Vitiligo Patients: A Case-Control Study
}

\author{
Jia-Wei Liu \\ Yan Tan \\ Tian Chen \\ Wei Liu \\ Yue-Tong Qian \\ Dong-Lai Ma
}

Department of Dermatology, Peking Union Medical College Hospital, Chinese Academy of Medical Sciences and Peking Union Medical College, National Clinical Research Center for Skin and Immune Diseases, Beijing, 100730, People's Republic of China
Correspondence: Dong-Lai Ma Department of Dermatology, Peking Union Medical College Hospital, Chinese Academy of Medical Sciences and Peking Union Medical College, National Clinical Research Center for Skin and Immune

Diseases, Beijing, 100730, People's Republic of China

Tel +86- $10-69151543$

$\mathrm{Fax}+86-10-69151502$

Email mdonglai@sohu.com
Background: Vitiligo can cause disfiguration, impair the social function of the patients and induce physiological burdens. However, limited research about the health-related quality of life has been conducted in vitiligo patients' sleeping conditions.

Objective: To evaluate the prevalence, severity, and risk factors of insomnia in vitiligo patients.

Methods: This case-control study was performed in March 2021. An online survey questionnaire including baseline information and the sleep-related instrument was sent to 762 vitiligo patients. The vitiligo-related evaluation was conducted by online video interview. According to whether having insomnia or not, patients were grouped and compared their clinical and demographic characteristics. The logistic regression model was conducted to analyze the risk factors for insomnia.

Results: A total of 409 patients were included. About $49.9 \%$ of patients (204/409) experienced insomnia. About 55.9\% (114/204) of the insomnia in vitiligo patients was adjustment sleep disorder caused by vitiligo. Development, aggravation, or recurrence of vitiligo were deemed as the first reason for insomnia in $71.1 \%$ of the sample $(81 / 114)$. There were significant differences in age $(32.1 \pm 4.1$ vs $27.9 \pm 4.2$ years, $\mathrm{P}<0.001)$, the percentage of female $(62.8 \%$ vs $49.3 \%, \mathrm{P}=0.006)$ and working in the urban areas $(77.0 \%$ vs $66.3 \%, \mathrm{P}=$ $0.017)$, vitiligo in face and neck $(67.2 \%$ vs $48.8 \%, \mathrm{P}<0.001)$, progression in vitiligo $(65.7 \%$ vs $49.3 \%, \mathrm{P}=0.001)$, oral corticosteroids $(25.0 \%$ vs $16.6 \%, \mathrm{P}=0.036)$ and depression $(5.4 \%$ vs $0.5 \%, \mathrm{P}=0.003$ ) between groups. After adjusting for gender, age and comorbidity, the multivariate logistic regression revealed that vitiligo in face and neck $(\mathrm{OR}=2.62 ; \mathrm{P}=0.032)$, progression in vitiligo $(\mathrm{OR}=2.50 ; \mathrm{P}=0.002)$, and oral corticosteroids $(\mathrm{OR}=2.71 ; \mathrm{P}=0.021)$ remained risk factors for insomnia in vitiligo patients.

Conclusion: Insomnia is prevalent in vitiligo patients. Dermatologists should identify this condition carefully, especially humanistic factors in social life, and perform individualized "non-drug" treatment.

Keywords: vitiligo, sleep disorder, insomnia, risk factors, insomnia severity index, psychodermatology

\section{Introduction}

Insomnia is becoming a more prominent public health problem in recent society and causes a social-economic burden. ${ }^{1}$ The annual prevalence of insomnia disorder ranges from $12 \%$ to $20 \%$ in the adult population. ${ }^{1}$ The onset of insomnia is often triggered by a specific life event, including losing a job or getting a disease, resulting in impairment of daytime function and distressful emotion. ${ }^{2}$ 
Vitiligo is the most common depigmentation dermatosis, affecting $0.5 \%$ to $2.0 \%$ of the global population. ${ }^{3}$ The disease can cause disfiguration, impair the social function of the patients and induce physiological burdens. The autoimmune hypothesis is widely accepted as the etiopathogenesis of vitiligo. ${ }^{4}$ Vitiligo is associated with various autoimmune diseases, such as autoimmune thyroid disease, alopecia areata, and type 1 diabetes mellitus. ${ }^{5}$ Moreover, physiological disorders show a higher prevalence in vitiligo patients compared with normal population, including depression, stress, and insomnia. ${ }^{6,7}$ In the meantime, various autoimmune diseases are associated with sleep disorders. ${ }^{8}$ Patients with sleep disorders may suffer the potential risk for vitiligo. ${ }^{9}$ Therefore, a bidirectional relationship exists between vitiligo and sleep disorders. The onset of vitiligo may be a causal trigger of sleeping disorders, while sleeping disorder may also induce or further aggravate vitiligo. Limited research about the health-related quality of life has been conducted in the sleeping condition of vitiligo patients. In this study, We conducted a telemedicine survey on insomnia in vitiligo patients through mobile phones. The study aims to evaluate the prevalence, severity, and risk factors of insomnia in vitiligo patients.

\section{Methods}

\section{Patients}

This case-control study was performed in March 2021. The study's research protocol was reviewed and approved by the Ethics Committee of the author's affiliation (Ethical No: S-K1587) following the Declaration of Helsinki. All participants were informed about the purpose and used tools of this research, and patients' written consents were obtained.

A total of 762 vitiligo patients who were under the follow-up by Prof. Ma were randomly picked from a pool of 3000 follow-up patients. Patients were excluded if he or she: (1) was under 18-year-old, (2) was not willing to participate in this study, (3) did not respond to the questionnaire before the deadline, (4) did not provide valid data, (5) was not willing to participate the online video interview, (6) did not understand the questions in the lists due to cognitive impairment or failure to recall the sleeprelated details during the onset of vitiligo, (7) had significant stressors or adverse life events such as bankruptcy, death of close relatives and severe earthquake, (8) had other categories of sleep disorders, such as obstructive sleep apnea syndrome, narcolepsy-cataplexy syndrome, circadian rhythm sleep disorders (eg, jet lag, shift work disorder, etc.), and parasomnias (eg, partial arousal disorders, REM behavior disorder, etc.). Finally, a total of 409 patients were included in this study. Demographic data and baseline information are detailed in Table 1 .

\section{Procedure}

We designed an online survey questionnaire that included the baseline information and a 45-item sleep-related instrument. The questionnaire was sent to the vitiligo patients through a social network application (WeChat). All the enrolled patients were invited to have an online video interview about the assessment of involved area and spreading of vitiligo lesion, using the instrument of Vitiligo Disease Activity (VIDA) and Vitiligo Area Scoring Index (VASI). The flow chart of this study is shown in Figure 1.

Table I Basic Information of the Included Patients

\begin{tabular}{|c|c|}
\hline Clinical Parameters & Values \\
\hline Age (Years, Mean \pm SD) & $30.0 \pm 4.5$ \\
\hline Gender (Female, n [\%]) & $229(56.0)$ \\
\hline Education ( $\geq$ Bachelor, $\mathrm{n}[\%]$ ) & $215(52.6)$ \\
\hline Workplace (Urban, n [\%]) & $293(71.6)$ \\
\hline Smoking (n [\%]) & $40(9.8)$ \\
\hline Alcohol (n [\%]) & $74(18.1)$ \\
\hline Duration of vitiligo( $\geq 3$ years, $n[\%])$ & 199(48.7) \\
\hline Area of vitiligo (VASI $\geq 6 \%, n[\%])$ & $34(8.3)$ \\
\hline Exposure area (Face and neck, $\mathrm{n}[\%])$ & $237(57.9)$ \\
\hline Insomnia (n [\%]) & 204(49.9) \\
\hline \multicolumn{2}{|l|}{ Progress of vitiligo } \\
\hline Progression & $235(57.5)$ \\
\hline Stability/Regression & $174(42.5)$ \\
\hline \multicolumn{2}{|l|}{ Treatments } \\
\hline Oral glucocorticoid & $85(20.8)$ \\
\hline Glycyrrhiza & $266(65.0)$ \\
\hline External ointment & $319(78.0)$ \\
\hline Masking agent & $29(7.1)$ \\
\hline Psoralen & $33(8.1)$ \\
\hline Phototherapy & $135(33.0)$ \\
\hline \multicolumn{2}{|l|}{ Comorbidity } \\
\hline Hypertension (n, \%) & $7(1.7)$ \\
\hline Cardiac diseases (n, \%) & $4(1.0)$ \\
\hline Diabetes mellitus ( $\mathrm{n}, \%)$ & $4(1.0)$ \\
\hline Hyperthyroidism (n, \%) & $31(7.6)$ \\
\hline Depression (n, \%) & $12(2.9)$ \\
\hline
\end{tabular}




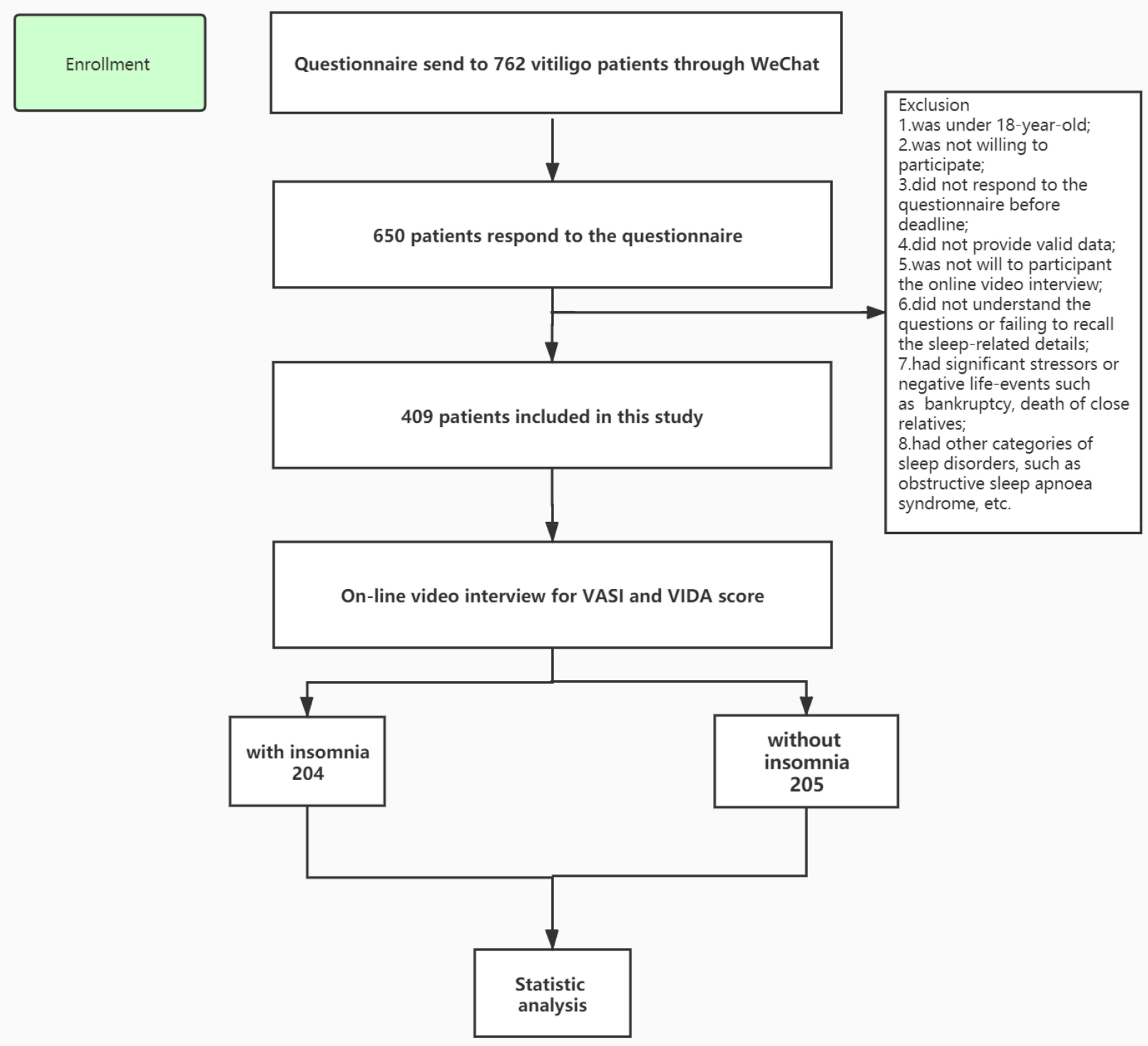

Figure I The flow chart of this study.

\section{Instruments \\ Vitiligo-Related Evaluation}

VIDA score was used to evaluate vitiligo activity by online video interview. It is a six-point scale based on the patient's report about disease activity. VIDA score was graded as follows: disease progression (expansion of existing lesions or appearance of new lesions) in 6 weeks or less, +4 ; disease progression in 6 weeks to 3 months, +3 ; disease progression in 3-6 months, +2 ; disease progression in 6-12 months, +1 ; stable for 1 year or more, +0 ; stable with spontaneous repigmentation for 1 year or more, $-1 .^{10}$
Involved area of vitiligo was assessed by VASI on the online video interview. VASI $=\sum$ [HAND UNITS $] \times$ [RESIDUAL DEPIGMENTATION]. ${ }^{11}$

\section{Insomnia-Related Evaluation}

The sleep-related instrument mainly included five parts: 1 . The insomnia diagnosis, 2. The Insomnia Severity Index, 3. The specific insomnia type, 4. Instruments and history requisition to exclude other sleep disorders, 5. Specific disturbance factors.

Among various sleep disorders, only insomnia was investigated in the present study. Insomnia was diagnosed 
according to the Diagnostic and Statistical Manual of Mental Disorders, Fifth Edition (DSM-V). Patients were asked about their forepassed sleep condition, including whether they are satisfied, whether they felt having trouble sleeping associated with impairment to daytime functioning or well-being, whether sleep inadequacy occurred three or more nights per week, and whether the sleep disturbance was associated with vitiligo.

Patients who reported inadequate sleep would fill the Insomnia Severity Index (ISI), a brief, reliable, validated self-reporting instrument that yields a quantitative index of perceived insomnia severity. The indicator is composed of 7 items. Sleep disturbance severity, sleep dissatisfaction, and the degree of daytime functional impairment, impairment perception and distress, and distress related to the sleeping problem were assessed. A final score higher than 8 is identified as having insomnia. A higher score means more severe insomnia. ${ }^{12}$

Besides, structured questions were asked to confirm the specific insomnia type: difficulty initiating sleep, difficulty in maintaining sleep, early morning wake up, or nonrestorative sleep.

Other sleep disorders such as obstructive sleep apnea, restless leg, circadian rhythm sleep disorders, narcolepsy, and parasomnias were ruled out by relative screening instruments (International restless legs scale, StopBANG sleep apnea questionnaire) and history inquiry (Did you fall asleep in the daytime at the "wrong occasion" and "wrong location?"; Did you have recurrent severe nightmares or dream walk?).

The following 8 subtypes of insomnia are recognized by ICSD-2. ${ }^{13}$

Adjustment insomnia. This refers to insomnia that is triggered or aggravated by an identifiable stressor (vitiligo in the present study).

Psychophysiological insomnia. This insomnia subtype is characterized by heightened arousal and learned sleeppreventing associations such as trying too hard to fall asleep or excessive worrying about sleep.

Insomnia due to mental disorder. This subtype can be diagnosed when patients having a definitive diagnosed mental disorder and insomnia constitute an unusually predominant portion of symptoms in the underlying mental disorder or when insomnia warrants independent, clinical attention.

Inadequate sleep hygiene. This type of insomnia is caused by habitual activities that result in poor sleep, including excessive daytime napping, caffeine or alcohol intake before bedtime, excessively stimulating activities close to bedtime, etc.

Idiopathic insomnia. This is a form of chronic insomnia that started in infancy or childhood, has no identifiable precipitant, and is chronic and relentless, with no periods of sustained remission. An imbalance in the neurological/ neurochemical sleep/wake system has been postulated.

Insomnia due to a medical condition. This is involved when a condition such as asthma or chronic heart failure can lead to insomnia.

Insomnia due to drugs or substances. This indicates that insomnia is based on the use of or withdrawal from prescription or recreational drugs, or it may be caused by food items or toxins such as carbon monoxide poisoning.

Physiological (organic) insomnia, unspecified. This is the category to use when insomnia does not fit into any of the insomnias mentioned before or when there is insufficiency of evidence to classify the patient into any of the above disorders.

\section{Statistical Analysis}

Data were analyzed using SPSS version 22 (IBM Corp., Armonk, NY, USA). Continuous variables were demonstrated as mean \pm standard deviation (SD) and ordinal variables as proportions. Student's $t$-test was performed to test the differences between groups in continuous variables, while Fisher exact test or Pearson chi-square test in ordinal variables. The Pearson correlation coefficient (coefficient, r) or Spearman's rank correlation (coefficient, rho) was performed to determine the correlation between ISI score and VASI and between ISI score and VIDA score controlling the parameters with a correlation value of more than 0.5 . The univariate logistic regression analysis was used to investigate the risk factors by calculating the odds ratio (OR) and $95 \%$ confidence interval (CI). The significant variables in the univariate logistic regression were included in multivariate analysis after adjusting the confounding factors. The cut-off points of the power analysis and statistical significance were $p$-value $\leq 0.8$ and 0.05 , respectively.

\section{Result}

About $56.0 \%$ of the included patients were women, and the mean $( \pm \mathrm{SD})$ age of the sample was $30.0( \pm 4.5)$ years. Table 1 shows their clinical and demographic characteristics. About $49.9 \%$ of patients (204/409) had an inadequate sleep, and 8.3\% (17/204) used sleeping pills regularly.

The prevalence of difficulty in falling asleep, difficulty in staying asleep, non-restorative sleep, and too early 
awakening that occurred 3 days per week was $65.2 \%$, $63.2 \%, 47.5 \%$, and $37.2 \%$, respectively.

\section{Subtypes of Insomnia}

About $87.3 \%$ of the sample (178/204) was deemed primary insomnias, while $12.7 \%$ was secondary insomnias. About 55.9\% (114/204) of insomnia in vitiligo was adjustment sleep disorder as the first reason, followed by psychophysiological insomnia $(13.7 \%, 28 / 204)$ and insomnia $(8.3 \%, 17 / 204)$ due to mental disorder. Specific makeup is shown in Table 2.

\section{Specific Analysis for the Vitiligo-Related Insomnia}

Several factors can result in sleep disturbance in insomnia in vitiligo patients. The specific order of precedence of the results in response to "please list the factors discounting your sleep quality" was shown in Table 3.

Among the vitiligo patients with the adjustment sleep disorder, development, aggravation, or recurrence of vitiligo was deemed as the first reasons for primary insomnia in

Table 2 Incidence of Subtypes of Insomnia

\begin{tabular}{|c|c|}
\hline Insomnias & $\begin{array}{l}\text { Number and Percentage of } \\
\text { Patients ( }[\%] \text { ) }\end{array}$ \\
\hline \multicolumn{2}{|l|}{ Primary insomnias } \\
\hline Adjustment sleep disorder & II 4 (55.9) \\
\hline Psychophysiological insomnia & $28(13.7)$ \\
\hline $\begin{array}{l}\text { Insomnia due to mental } \\
\text { disorder }\end{array}$ & $17(8.3)$ \\
\hline Inadequate sleep hygiene & $13(6.4)$ \\
\hline Idiopathic insomnia & $6(2.9)$ \\
\hline Total percentage & I $78(87.3)$ \\
\hline \multicolumn{2}{|l|}{ Secondary insomnias } \\
\hline $\begin{array}{l}\text { Insomnia due to medical } \\
\text { condition }\end{array}$ & $14(6.9)$ \\
\hline $\begin{array}{l}\text { Insomnia due to drug or } \\
\text { substance }\end{array}$ & $5(2.5)$ \\
\hline $\begin{array}{l}\text { Physiological (organic) } \\
\text { insomnia }\end{array}$ & $4(2.0)$ \\
\hline $\begin{array}{l}\text { Others (such as lactation, } \\
\text { ambient noise) }\end{array}$ & $3(1.5)$ \\
\hline Total percentage & $26(12.7)$ \\
\hline
\end{tabular}

$71.1 \%$ of the sample $(81 / 114)$, followed by appearance $(67.5 \%, 77 / 114)$ and working/campus life $(48.2 \%, 55 / 114)$.

\section{Comparisons Between Insomnia Patients and Non-Insomnia Patients with Vitiligo}

There were significant differences in age $(32.1 \pm 4.1$ vs 27.9 \pm 4.2 years, $\mathrm{P}<0.001)$, the percentage of female $(62.8 \% \mathrm{vs}$ $49.3 \%, \mathrm{P}=0.006)$ and working in the urban areas $(77.0 \%$ vs $66.3 \%, \mathrm{P}=0.017)$, vitiligo in face and neck $(67.2 \%$ vs $48.8 \%, \mathrm{P}<0.001)$, progression in vitiligo $(65.7 \%$ vs $49.3 \%, \mathrm{P}=0.001)$, oral corticosteroids $(25.0 \%$ vs $16.6 \%$, $\mathrm{P}=0.036)$ and depression $(5.4 \%$ vs $0.5 \%, \mathrm{P}=0.003)$ between groups (Table 4).

\section{Risk Analysis for Insomnia Patients in Vitiligo}

The univariable logistic regression demonstrated that age $\geq 30$ years $(\mathrm{OR}=1.85 ; 95 \% \mathrm{CI}: 1.19-2.73 ; \mathrm{P}=0.006)$, female $(\mathrm{OR}=1.52 ; 95 \% \mathrm{CI}: 1.00-2.31 ; \mathrm{P}=0.048)$, vitiligo in face and neck $(\mathrm{OR}=2.32 ; 95 \% \mathrm{CI}: 1.76-2.87 ; \mathrm{P}=0.012)$, progression in vitiligo ( $\mathrm{OR}=2.10 ; 95 \% \mathrm{CI}: 1.23-2.92 ; \mathrm{P}=0.023)$, oral corticosteroids ( $\mathrm{OR}=2.41 ; 95 \%$ CI:1.65-3.03; $\mathrm{P}=0.013)$ and depression $(\mathrm{OR}=2.52 ; 95 \% \mathrm{CI}: 1.92-2.93 ; \mathrm{P}=0.001)$ were risk factors for insomnia patients in vitiligo.

After adjusting for gender, age and comorbidity, the multivariate logistic regression revealed that vitiligo in face and neck $(\mathrm{OR}=2.62 ; 95 \% \mathrm{CI}: 2.03-3.17 ; \mathrm{P}=0.032)$, progression in vitiligo $(\mathrm{OR}=2.50 ; 95 \% \mathrm{CI}: 1.62-3.31$; $\mathrm{P}=0.002)$, and oral corticosteroids $\quad(\mathrm{OR}=2.71 ; 95 \%$ CI:2.11-3.19; $\mathrm{P}=0.021$ ) remained risk factors for insomnia patients in vitiligo (Table 5).

\section{Correlations Analysis}

There was no significant correlation between ISI score and VASI ( $\mathrm{r}=0.502, \mathrm{R} 2=0.25, \mathrm{P}=0.32$ ). But a significant correlation was identified between ISI score and VIDA score (rho $=0.620, \mathrm{R} 2=0.38, \mathrm{P}<0.01$ ).

\section{Discussion}

Various studies have evaluated the association between sleep problems and dermatoses, such as atopic dermatitis and psoriasis. ${ }^{14-16}$ These disorders are closely related to either apparent symptoms such as itch and pain, ${ }^{17}$ or multiple comorbidities including obesity, metabolic syndrome, hypertension, diabetes, cardiovascular events, asthma, and psychiatric disorders, which can all affect the course of sleep. ${ }^{14,18}$ However, little attention has 
Table 3 Specific Analysis for the Vitiligo-Related Insomnia (Adjustment Sleep Disorder)

\begin{tabular}{|c|c|c|}
\hline Factors & $\begin{array}{l}\text { Number and Percentage } \\
\text { of Patients ( }[\%])\end{array}$ & Examples \\
\hline $\begin{array}{l}\text { Development, aggravation, or } \\
\text { recurrence of vitiligo }\end{array}$ & $81(71.1)$ & $\begin{array}{l}\text { Concern about the development, aggravation or reoccur of vitiligo, which may } \\
\text { result in constant nightmares }\end{array}$ \\
\hline Appearance & $77(67.5)$ & $\begin{array}{l}\text { Physiologically devastating of disfigurement caused by vitiligo which makes them } \\
\text { have a singular appearance }\end{array}$ \\
\hline Working/campus life & $55(48.2)$ & $\begin{array}{l}\text { Experienced discriminative or unpleasant event due to vitiligo in working or } \\
\text { campus life }\end{array}$ \\
\hline Fertility problems & $54(47.4)$ & Concern about the inheritance tendency of vitiligo, hesitate about childbearing \\
\hline $\begin{array}{l}\text { Non-working/ recreational } \\
\text { activities }\end{array}$ & $45(39.5)$ & $\begin{array}{l}\text { Feel embarrassed when changing clothes or expose affected body areas during } \\
\text { non-working activities }\end{array}$ \\
\hline Financial burden & $42(36.8)$ & $\begin{array}{l}\text { Medical expenses including medicines, camouflage cosmetics, and traffic expenses, } \\
\text { etc }\end{array}$ \\
\hline $\begin{array}{l}\text { Side effects from vitiligo- } \\
\text { related drugs }\end{array}$ & $32(28.1)$ & $\begin{array}{l}\text { Worry about other side-effect caused by steroid such as weight gain, or acne } \\
\text { eruption; }\end{array}$ \\
\hline $\begin{array}{l}\text { Marital relationship or other } \\
\text { intimate relationship }\end{array}$ & $31(27.2)$ & $\begin{array}{l}\text { Vitiligo affect intimate relationship, such as discounted sexual intercourse in } \\
\text { marriage bonds, loss of confidence in front of their children }\end{array}$ \\
\hline
\end{tabular}

been paid to the relationship between vitiligo and inadequate sleep. As the most common depigmentation dermatosis, vitiligo causes disfiguration to impair the patients' physiological well-being and results in negative emotions such as anxiety and depression, which may interfere with sleep. ${ }^{19}$ The present findings demonstrated that up to $49.9 \%$ of vitiligo patients (204/409) had inadequate sleep. $55.9 \%$ of the vitiligo patients with insomnia reported the adjustment sleep disorder resulting from the vitiligo. Therefore, vitiligo-induced insomnia is prevalent. After adjusting for gender, age and comorbidity, the multivariate logistic regression revealed that vitiligo in the face and neck $(\mathrm{OR}=2.62)$, progression in vitiligo $(\mathrm{OR}=2.50)$, and oral corticosteroids $(\mathrm{OR}=2.71)$ were the risk factors for insomnia in vitiligo patients.

To the best of our knowledge, how and why failing to sleep well in patients with vitiligo has never been conducted in a previous study. In 2008, Mouzas et al investigated the relationship between vitiligo and sleep disorders. ${ }^{20}$ However, this study focused specifically on parasomnias. Its findings showed that patients suffering from vitiligo reported a significantly higher occurrence of sleepwalking, nocturnal enuresis, night illusions, sleep terrors, and nightmares than that of the control group before the occurrence of the disease. The authors assumed that association might exist between parasomnias in early life and subsequent onset of vitiligo, and neural mechanisms involving monoaminergic systems (especially the serotoninergic one) might potentially play an important role in the pathological mechanism of vitiligo. In the present study, we ranked the main subtypes of insomnia in vitiligo patients and analyzed its causes. Among the vitiligo patients with insomnia, development, aggravation, or recurrence of vitiligo was deemed the first reason for primary insomnia, followed by appearance and working/ campus life. These figures further supported the onset of vitiligo as a psychological stressor that resulted in insomnia. Besides, a percentage ranging from $36.8 \%$ to $47.4 \%$ reported the concern about the medical cost of treating this disease, limitation in recreational activities due to significant stigma and concern about the inheritance of vitiligo. Despite a lower incidence of reporting side effects from vitiligo-related drugs and marital relationships or other intimate relationships than other reasons, it is not rare. It remains to be paid attention to from dermatologists. Collectively, vitiligo is frequently associated with social stigma and stressors. As a chronic illness lasting several decades and even throughout an entire lifetime, the stress may accumulate and result in insomnia, finally compromising the normal circadian rhythms. Vandeputte et al revealed that patients with chronic sleep disorders are prone to depression. ${ }^{21}$ Numerous studies have 
Table 4 Comparisons Between Insomnia Patients and Non-Insomnia Patients in Vitiligo

\begin{tabular}{|c|c|c|c|}
\hline Clinical Parameters & Insomnia Patients $(n=204)$ & Non-Insomnia Patients $(n=205)$ & $P$ value \\
\hline Age (Years) & $32.1 \pm 4.1$ & $27.9 \pm 4.2$ & $<0.001 *$ \\
\hline Gender (Female, n [\%]) & $128(62.8)$ & $101(49.3)$ & $0.006 *$ \\
\hline Education ( $\geq$ Bachelor, $\mathrm{n}[\%]$ ) & $97(47.5)$ & II 8 (57.6) & 0.851 \\
\hline Workplace (Urban, n [\%]) & $157(77.0)$ & $136(66.3)$ & $0.017^{*}$ \\
\hline Smoking (n [\%]) & $24(11.8)$ & $16(7.8)$ & 0.120 \\
\hline Alcohol (n [\%]) & $42(20.6)$ & $32(15.6)$ & 0.191 \\
\hline Duration of vitiligo ( $\geq 3$ years, $n[\%]$ ) & $101(49.5)$ & $98(47.8)$ & 0.806 \\
\hline Area of vitiligo (VASI $\geq 6 \%, n$ [\%]) & $19(9.3)$ & $15(7.3)$ & 0.581 \\
\hline Exposure area (Face and neck, n [\%]) & $137(67.2)$ & $100(48.8)$ & $<0.001 *$ \\
\hline \multicolumn{4}{|l|}{ Progress of vitiligo } \\
\hline Progression & 134(65.7) & $101(49.3)$ & $0.001 *$ \\
\hline Stability/Regression & $70(34.3)$ & 104(50.7) & \\
\hline \multicolumn{4}{|l|}{ Treatments } \\
\hline Oral corticosteroids & $5 \mathrm{I}(25.0)$ & $34(16.6)$ & $0.036 *$ \\
\hline Glycyrrhiza & $136(66.7)$ & $130(63.4)$ & 0.558 \\
\hline Topical steroid/calcineurin ointment & $153(75.0)$ & $166(81.0)$ & 0.181 \\
\hline Camouflage agent & $14(6.9)$ & $15(7.3)$ & 0.858 \\
\hline Psoralen & $17(8.3)$ & $16(7.8)$ & 0.844 \\
\hline Phototherapy & $71(34.8)$ & $64(31.2)$ & $0.44 I$ \\
\hline \multicolumn{4}{|l|}{ Comorbidity } \\
\hline Hypertension (n, \%) & $2(1.0)$ & $5(2.4)$ & 0.255 \\
\hline Cardiac diseases (n, \%) & $3(1.5)$ & $\mathrm{I}(0.5)$ & 0.313 \\
\hline Diabetes mellitus (n, \%) & $2(1.0)$ & $2(1.0)$ & 0.996 \\
\hline Hyperthyroidism (n, \%) & $14(6.9)$ & $17(8.3)$ & 0.585 \\
\hline Depression (n, \%) & II (5.4) & I (0.5) & $0.003^{*}$ \\
\hline
\end{tabular}

Note: *Means significantly different between groups.

demonstrated the depression adversely affecting the quality of life of patients with vitiligo. ${ }^{22-24}$ Given this, there is a vicious circle between vitiligo and depression. However, in such a pathological process, inadequate sleep is seemingly intermediate between vitiligo and depression. Of note, the difference between adjustment sleep disorder and psychophysiological insomnia should be clarified. The former has definite stressor, and the latter is a type of conditioned or learned insomnia that involves either the conditioning of sleep-preventing habits in which repeated

Table 5 Analysis of Risk Factors for Vitiligo Patients with Insomnia

\begin{tabular}{|c|c|c|c|c|}
\hline \multirow[t]{2}{*}{ Variables } & \multicolumn{2}{|c|}{ Univariate } & \multicolumn{2}{|c|}{ Multivariate } \\
\hline & OR (95\% CI) & $P$ value & $O R^{\#}(95 \% \mathrm{Cl})$ & $P$ value \\
\hline Age (years) $\geq 30$ & $1.85(1.19-2.73)$ & $0.006^{*}$ & - & - \\
\hline Female & $1.52(1.00-2.31)$ & $0.048^{*}$ & - & - \\
\hline Vitiligo in face and neck & $2.32(1.76-2.87)$ & $0.012^{*}$ & $2.62(2.03-3.17)$ & $0.032^{*}$ \\
\hline Progression in vitiligo & $2.10(1.23-2.92)$ & $0.023^{*}$ & $2.50(1.62-3.31)$ & $0.002 *$ \\
\hline Oral corticosteroids & $2.4 I(1.65-3.03)$ & $0.013^{*}$ & $2.71(2.11-3.19)$ & $0.021 *$ \\
\hline Depression & $2.52(1.92-2.93)$ & $0.00 I^{*}$ & - & - \\
\hline
\end{tabular}

Notes: *Indicates statistical significance; ${ }^{*}$ adjusted for age, gender and comorbidity; Odds ratios, as well as $95 \%$ Cls, were shown.

Abbreviations: $\mathrm{OR}$, odds ratio; $\mathrm{Cl}$, confidence interval. 
impairing of sleeplessness and situational, temporal, or behavioral stimuli normally associated with sleep leads to conditional arousal that impairs sleep, or somatized tension believed to result from the internalization of psychological conflicts and excessive worrying about sleep, which are incompatible with sleep. ${ }^{25,26}$ Simply, both adjustment sleep disorder and psychophysiological insomnia appear to result from psychological factors. However, the former lies in vitiligo's stress, but the latter is caused by excessive concentration on sleep problems. In comparison with the rest of insomnia such as those due to mental disorder (eg, bipolar disorder), inadequate sleep hygiene (eg, coffee or tea), with a lower incidence, the present figure strongly indicated that adjustment sleep disorder and psychophysiological insomnia, especially for the former one, vitiligo-induced insomnia, is a prevalent problem among patients with vitiligo.

Significant differences in age, the percentage of females working in the urban areas, vitiligo in face and neck, progression in vitiligo, oral corticosteroids, and depression between the two groups were found in the present findings. Moreno et al demonstrated female gender had a higher prevalence of sleep disorders. ${ }^{27}$ Sidani et al indicated gender differences in insomnia could be associated with women's stress, expression of somatic symptoms, and interest in maintaining their health to meet multiple role demands. ${ }^{28}$ These elements, either isolated or combined, can compromise sleep quality. Besides, an experimental study demonstrated that sleep regulation in female mice was dementedly affected by hormonal variations associated with the estrous cycle caused by sex hormone. $^{29}$ Nam et al found a significant association between sleep duration and menstrual cycle in female adolescents. $^{30}$ This may help explain why female vitiligo patients reported insomnia more frequently. In this study, the vitiligo patients with insomnia were older in age compared to those without insomnia. This is consistent with previous studies. $^{31,32}$ After adjusting for gender, age, and comorbidity, the multivariate logistic regression revealed that vitiligo in the face and neck and vitiligo progression were risk factors for insomnia in vitiligo patients. Even though vitiligo does not cause systemic symptoms, the effect vitiligo has on quality of life is similar to other skin diseases, such as psoriasis and atopic dermatitis. ${ }^{4}$ Its impact on quality of life is mainly associated with negative psychological influence, for example, reduced self-esteem, increased embarrassment. These elements negatively affect social or sexual relations, resulting in vitiligo- related isolation, discrimination, social stigma, and even suicidal ideation. ${ }^{33-36}$ These feelings, which become a barrier for campus life, seeking a job, and even marriage directly or indirectly, would constantly exist in patients with vitiligo and thus impaired sleep quality. As for oral corticosteroids, previous studies have demonstrated steroids reduced REM sleep and tended to enhance intermittent wakefulness. ${ }^{37,38}$ This may be related to the effect of corticosteroids on the function of pituitary-adrenal and pituitary-thyroid. Moreover, administration of oral steroids is usually associated with rapid disease progression in clinical practice. ${ }^{39}$ The disease exacerbation may cause physiological stress and subsequently result in insomnia.

The present study has demonstrated that insomnia was a prevalent condition in our sample. Seemingly, this sleep problem affected their quality of life by a psychopathological condition such as depression and stress. Therefore, it may be significant for dermatologists to have general identification of these conditions in vitiligo patients, both psychological and physical, and offer individualized therapy. Not only routine treatment but also the evaluation for the psychiatric status of patients with vitiligo, especially women and the elderly, should be considered. Besides, the interaction between the patient and the dermatologists is also crucial. In some cases with severe sleep problems, the psychiatrist-patients communication may be more significant. Papadopoulos et al demonstrated that effective counseling could contribute to improving self-esteem and quality of life in vitiligo patients and may even positively influence the course of this chronic condition. ${ }^{40}$ Till now, no relevant study has been conducted between insomnia treatment and vitiligo disease control. However, theoretically, improved sleep quality may have a positive influence on the disease course. Moreover, in patients with other comorbidities such as heart diseases treated with beta-blockers, treatment of insomnia can also ease the physiological burden, deactivate the hypothalamus-pituitary-adrenergic axis, decrease the excitability of the sympathetic nervous system, and result in better control of skin dermatosis and systemic disorders. From the psychological effect on sleep disorders, the patients' quality of life can be significantly improved. Of note, different patients with vitiligo have specific worries about this disease and problems. Dermatologists should identify them carefully, especially humanistic factors in social life, and perform individualized "non-drug" treatment. 
There are several limitations in this research. First, only insomnia was investigated. However, in addition to insomnia, the international classification of sleep disorders lists another seven categories of sleep disorders, such as obstructive sleep apnoea syndrome, narcolepsy-cataplexy syndrome, etc. Second, the evaluating method for a sleep disorder was subjective instead of objective methods such as polysomnography (PSG). This may not benefit the reliable conclusions. The detailed information about insomnia may be easily affected by the patient's psychological factors such as cognitive processes and recall bias. A discrepancy between subjective reporting and objective assessments about the disrupted sleep exists. Especially considering the disease, with a course of a long slow continuous progress, parts of included patients with vitiligo failed to report the actual situation of insomnia when they just had the vitiligo. Thirdly, image clarity was limited by mobile phone camera resolution. Diminution of accuracy in VASI evaluation may exist during online interview. Last, the subtypes and reasons for insomnia in vitiligo patients in the present Tables 2 and 3 may not list the whole picture. We could neglect insidious ones behind this chronic disorder with complex psychological activities. These limitations open the door for the further study.

\section{Conclusion}

Vitiligo can cause disfiguration, impair the social function of the patients and induce physiological burdens. The present findings revealed that vitiligo in face and neck, progression in vitiligo, and oral corticosteroids were risk factors for insomnia in vitiligo patients. Therefore, insomnia is prevalent in vitiligo patients. Dermatologists should identify this condition carefully, especially humanistic factors in social life, and perform individualized "non-drug" treatment.

\section{Acknowledgment}

We would like to thank all patients involved in this study. The patients in this manuscript have given written informed consent to publication of their case details.

\section{Funding}

The article had no funding source.

\section{Disclosure}

All authors report no conflict of interest.

\section{References}

1. Buysse DJ. Insomnia. JAMA. 2013;309:706-716. doi:10.1001/ jama.2013.193

2. Wilson S, Anderson K, Baldwin D, et al. British association for psychopharmacology consensus statement on evidence-based treatment of insomnia, parasomnias and circadian rhythm disorders: an update. $\quad J$ Psychopharmacol. 2019;33:923-947. doi:10.1177/ 0269881119855343

3. Ezzedine K, Eleftheriadou V, Whitton M, van Geel N. Vitiligo. Lancet. 2015;386:74-84. doi:10.1016/S0140-6736(14)60763-7

4. Rodrigues M, Ezzedine K, Hamzavi I, Pandya AG, Harris JE. New discoveries in the pathogenesis and classification of vitiligo. $\mathrm{J} \mathrm{Am}$ Acad Dermatol. 2017;77:1-13. doi:10.1016/j.jaad.2016.10.048

5. Elbuluk N, Ezzedine K. Quality of life, burden of disease, co-morbidities, and systemic effects in vitiligo patients. Dermatol Clin. 2017;35:117-128. doi:10.1016/j.det.2016.11.002

6. Simons RE, Zevy DL, Jafferany M. Psychodermatology of vitiligo: psychological impact and consequences. Dermatol Ther. 2020;33: e13418. doi:10.1111/dth. 13418

7. Sharma N, Koranne RV, Singh RK. Psychiatric morbidity in psoriasis and vitiligo: a comparative study. J Dermatol. 2001;28:419-423. doi:10.1111/j.1346-8138.2001.tb00003.x

8. Hsiao YH, Chen YT, Tseng CM, et al. Sleep disorders and increased risk of autoimmune diseases in individuals without sleep apnea. Sleep. 2015;38:581-586. doi:10.5665/sleep.4574

9. Seo HM, Kim TL, Kim JS. The risk of alopecia areata and other related autoimmune diseases in patients with sleep disorders: a Korean population-based retrospective cohort study. Sleep. 2018;41:1-8. doi:10.1093/sleep/zsy111

10. Njoo MD, Das PK, Bos JD, Westerhof W. Association of the Köbner phenomenon with disease activity and therapeutic responsiveness in vitiligo vulgaris. Arch Dermatol. 1999;135:407-413. doi:10.1001/ archderm.135.4.407

11. Hamzavi I, Jain H, McLean D, Shapiro J, Zeng H, Lui H. Parametric modeling of narrowband UV-B phototherapy for vitiligo using a novel quantitative tool: the Vitiligo Area Scoring Index. Arch Dermatol. 2004;140:677-683. doi:10.1001/archderm.140.6.677

12. Morin CM, Belleville G, Bélanger L, Ivers H. The Insomnia Severity Index: psychometric indicators to detect insomnia cases and evaluate treatment response. Sleep. 2011;34:601-608. doi:10.1093/sleep/ 34.5.601

13. Thorpy MJ. Classification of sleep disorders. Neurotherapeutics. 2012;9:687-701. doi:10.1007/s13311-012-0145-6

14. Gupta MA, Simpson FC, Gupta AK. Psoriasis and sleep disorders: a systematic review. Sleep Med Rev. 2016;29:63-75. doi:10.1016/j. smrv.2015.09.003

15. Reszke R, Szepietowski JC. End-stage renal disease chronic itch and its management. Dermatol Clin. 2018;36:277-292. doi:10.1016/j. det.2018.02.007

16. Bender BG, Ballard R, Canono B, Murphy JR, Leung DY. Disease severity, scratching, and sleep quality in patients with atopic dermatitis. J Am Acad Dermatol. 2008;58:415-420. doi:10.1016/j. jaad.2007.10.010

17. Kaaz K, Szepietowski JC, Matusiak $Ł$. Influence of itch and pain on sleep quality in atopic dermatitis and psoriasis. Acta Derm Venereol. 2019;99:175-180. doi:10.2340/00015555-3065

18. Bender BG, Leung DY. Sleep disorders in patients with asthma, atopic dermatitis, and allergic rhinitis. J Allergy Clin Immunol. 2005;116:1200-1201. doi:10.1016/j.jaci.2005.09.041

19. Hamidizadeh N, Ranjbar S, Ghanizadeh A, Parvizi MM, Jafari P, Handjani F. Evaluating prevalence of depression, anxiety and hopelessness in patients with Vitiligo on an Iranian population. Health Qual Life Outcomes. 2020;18:20. doi:10.1186/s12955-020-1278-7 
20. Mouzas O, Angelopoulos N, Papaliagka M, Tsogas P. Increased frequency of self-reported parasomnias in patients suffering from vitiligo. Eur J Dermatol. 2008;18:165-168.

21. Vandeputte M, de Weerd A. Sleep disorders and depressive feelings: a global survey with the Beck depression scale. Sleep Med. 2003;4:343-345. doi:10.1016/S1389-9457(03)00059-5

22. Lai YC, Yew YW, Kennedy C, Schwartz RA. Vitiligo and depression: a systematic review and meta-analysis of observational studies. Br J Dermatol. 2017;177:708-718. doi:10.1111/bjd.15199

23. Wang G, Qiu D, Yang H, Liu W. The prevalence and odds of depression in patients with vitiligo: a meta-analysis. J Eur Acad Dermatol Venereol. 2018;32:1343-1351. doi:10.1111/jdv.14739

24. Silpa-Archa N, Pruksaeakanan C, Angkoolpakdeekul N, et al. Relationship between depression and quality of life among vitiligo patients: a self-assessment questionnaire-based study. Clin Cosmet Investig Dermatol. 2020;13:511-520. doi:10.2147/CCID.S265349

25. Leistedt S, Kempenaers C, Linkowski P. Neurophysiological and clinical aspects of psychophysiological insomnia. Rev Med Brux. 2007;28:11-20.

26. Perlis ML, Giles DE, Mendelson WB, Bootzin RR, Wyatt JK. Psychophysiological insomnia: the behavioural model and a neurocognitive perspective. J Sleep Res. 1997;6:179-188. doi:10.1046/j.1365-2869.1997.00045.x

27. Moreno CRC, Santos JLF, Lebrão ML, Ulhôa MA, Duarte YAO. Sleep disturbances in older adults are associated to female sex, pain and urinary incontinence. Rev Bras Epidemiol. 2019;21(Suppl 02): e180018. doi:10.1590/1980-549720180018.supl.2

28. Sidani S, Guruge S, Fox M, Collins L. Gender differences in perpetuating factors, experience and management of chronic insomnia. J Gend Stud. 2019;28:402-413. doi:10.1080/09589236. 2018.1491394

29. Koehl M, Battle SE, Turek FW. Sleep in female mice: a strain comparison across the estrous cycle. Sleep. 2003;26:267-272. doi:10.1093/sleep/26.3.267

30. Nam GE, Han K, Lee G. Association between sleep duration and menstrual cycle irregularity in Korean female adolescents. Sleep Med. 2017;35:62-66. doi:10.1016/j.sleep.2017.04.009
31. Luca G, Haba Rubio J, Andries D, et al. Age and gender variations of sleep in subjects without sleep disorders. Ann Med. 2015;47:482-491. doi:10.3109/07853890.2015.1074271

32. Åkerstedt T, Ghilotti F, Grotta A, Bellavia A, Lagerros YT, Bellocco R. Sleep duration, mortality and the influence of age. Eur J Epidemiol. 2017;32:881-891. doi:10.1007/s10654-017-0297-0

33. Porter J, Beuf AH, Nordlund JJ, Lerner AB. Psychological reaction to chronic skin disorders: a study of patients with vitiligo. Gen Hosp Psychiatry. 1979;1:73-77. doi:10.1016/0163-8343(79)90081-1

34. Porter J, Beuf AH, Lerner A, Nordlund J. Response to cosmetic disfigurement: patients with vitiligo. Cutis. 1987;39:493-494.

35. Porter JR, Beuf AH, Lerner AB, Nordlund JJ. The effect of vitiligo on sexual relationships. J Am Acad Dermatol. 1990;22:221-222. doi:10.1016/0190-9622(90)70028-G

36. Pahwa P, Mehta M, Khaitan BK, Sharma VK, Ramam M. The psychosocial impact of vitiligo in Indian patients. Indian J Dermatol Venereol Leprol. 2013;79:679-685. doi:10.4103/03786323.116737

37. Fehm HL, Benkowitsch R, Kern W, Fehm-Wolfsdorf G, Pauschinger P, Born J. Influences of corticosteroids, dexamethasone and hydrocortisone on sleep in humans. Neuropsychobiology. 1986;16:198-204. doi:10.1159/000118326

38. Holsboer F, Wiedemann K, Rupprecht R, Steiger A. Effects of corticosteroids and neurosteroids on sleep EEG. Clin Neuropharmacol. 1992;15(Pt A):588a-589a. doi:10.1097/00002826199201001-00305

39. Pasricha JS, Khaitan BK. Oral mini-pulse therapy with betamethasone in vitiligo patients having extensive or fast-spreading disease. Int $J$ Dermatol. 1993;32:753. doi:10.1111/j.1365-4362.1993. tb02754.x

40. Papadopoulos L, Bor R, Legg C. Coping with the disfiguring effects of vitiligo: a preliminary investigation into the effects of cognitive-behavioural therapy. Br J Med Psychol. 1999;72(Pt 3):385-396. doi:10.1348/000711299160077

\section{Publish your work in this journal}

Clinical, Cosmetic and Investigational Dermatology is an international, peer-reviewed, open access, online journal that focuses on the latest clinical and experimental research in all aspects of skin disease and cosmetic interventions. This journal is indexed on CAS.
The manuscript management system is completely online and includes a very quick and fair peer-review system, which is all easy to use. Visit http://www.dovepress.com/testimonials.php to read real quotes from published authors. 